\title{
Spotlight on How to Structure a Case Report
}

\author{
Ario Santini ${ }^{1 *}$ \\ 1 University of Medicine, Pharmacy, Science and Technology of Târgu Mureș, Romania; Hon Fellow, University of \\ Edinburgh, United Kingdom
}

This is the first paper in the occasional series which aims to aid and facilitate internists and colleagues who have little or no experience in writing and submitting manuscripts for publication.

Case reports have a very low level of evidence, but they do have a place in medical publications. Polymyalgia rheumatica (PMR) was first established as a distinct disease in 1966 following the publication of a case report on 11 patients. [ Davison S, Spiera H, Plotz CM "Polymyalgia rheumatica". Arthritis and Rheumatism. 9 (1): 18-23.]

\section{WHAT IS A CASE REPORT?}

A case report is a means of communicating something new that has been learnt from clinical practice:

- An unusual or previously unknown condition

- A rare presentation or complication of a known disease

- A new approach to managing a common condition

\section{THE FIRST LINE OF EVIDENCE IN THE MEDICAL LITERATURE}

The literature is reviewed to enable the authors to understand the current issues regarding the type of case they are reporting and to be able to discuss these and any developments that they consider essential. However, the structure of a Case report should not include a literature review as such, which is a distinctly separate and more extended entity.

Once the authors are familiar with currently published papers, only the most pertinent should be quoted in a Case report, usually not more than five and less than ten. (How to write a Literature Review is the subject of a future paper).

Significant value in writing a Case report is that it provides medical students and junior doctors with an excellent opportunity to develop their writing skills.

\section{WHERE DOES ONE FIND CASES TO REPORT?}

Look for unusual cases encountered in clinics and wards. If something interesting is found, discuss it with the consultant in charge of the case. The idea must be novel or striking about a particular patient. Consultants may have unpublished data that can be used as the subject of a case report. They would probably be delighted if for some else to write up the case.

Remember the patient is under the care of a consultant; do not proceed without obtaining the appropriate authority. Moreover, it is usual to include the consultant's name on any submitted manuscript.

A Case report comprises the following segments:

- An Abstract

- An Introduction (this will include an aim or objective and reference to pertinent published literature)

- Case presentation

- A Discussion Section [including comments on the published literature]

- A Conclusion

- References

Title and keywords will also be necessary, and these are best decided on after the major segments of the manuscript have been completed.

However, it is advisable not to write the paper in this order. The following list outlines the order that is advisable:

\section{Case presentation}

First, tackle the details of the case. The case presentation is a fundamental part of the case report. Describe the case in detail and chronological order. Readers should be able to determine the validity of the case and the authors' conclusions from this report.

As a general rule use simple, concise and precise sentences, avoiding any unnecessary words or phrases. 
Maintain a strict focus on giving a chronological account of the case. Do not depart from the recorded facts of the case as detailed in the patient's records. Avoid speculating on underlying disease processes or treatments. Keep to the recorded facts.

The following should be addressed.

First, describe the patient's initial complaint and, if possible, use the patient's own words.

Describe why the patient has come to YOU or your Hospital and who referred him/her to the current hospital. Briefly describe the patient, gender, ethnicity his chief complaint, medical history, family and social history. If he has been seen by a previous doctor, who referred him to the hospital, briefly state what diagnosis had been arrived at and why the doctor considered it appropriate for him to be referred. Do not give excessive details of any previous routine tests or examinations.

Detail the tests performed on admission and any abnormalities found. This will be the baseline day to which will be referred to as treatment is progressed. All future tests and treatments should be described in a contributing and time-based relationship.

Record the effect of each test and treatment and any unexpected outcomes. Detail treatment changes and why they were undertaken. At this point in the description of the case, do not enter into a discussion of any part of the treatment or why tests were undertaken, or drug regimens altered or changed. Maintain a strictly factual account. Leave any discussion analysis to the Discussion section.

It is useful to include how and why treatment finished. Was it decided to terminate care, and if so, why? Did the patient withdraw from care or was he referred on to another hospital or specialist department or hospital? Whatever of these apply, record the patient's final outcome, when he was discharged and list any proposed ongoing treatments. Finally, give the patient's status at the time of the end report.

When writing the first draft of a manuscript, it is good practice to give concise, detailed answers to these points as "bullet points " in the first instance. Later they will be combined into flowing readable text.

\section{Introduction \& Discussion Sections}

These sections will be predicated on a previously undertaken literature search. The literature search is a critical step. If the findings have already been reported, publishing an article will be near impossible, so make sure that a thorough literature search has been performed using PubMed, Medline, Ovid, Embase and Google Scholar. Also, consult case report journals that are not indexed in PubMed, e.g. International Medical Case Reports Journal.

In the Introduction summarise the case report in an opening sentence. Then say how rare this condition is and why the case report is essential. It is of importance to give the reader a clear idea of what is interesting about the case. Start by placing the study in a historical or social context. If there have been similar previously reported cases, do not avoid mentioning them and describe briefly. Describe anything especially demanding difficult or thought-provoking about the diagnosis or management of the current disorder. Cite each previous study. An introduction should be no more than a few paragraphs long, with the overall objective is to have the reader understand clearly of the case.

The Discussion is the selling point of a case report requiring a well-written argument to convince an Editor and the reader that the case report is credible convincing and worthy of publication.

Start by briefly expanding on the introduction, explaining why the case is essential and why it will be of interest to the reader. Follow by summing up the current pertinent literature associated with the case. Describe previous reports on the topic.

Explain the clinical findings and in such a way that reviewers and readers will be convinced of evidence that the case is uncommon and atypical and perhaps rare if not unique.

\section{A Conclusion}

When the journal requires a brief conclusion or summary of learning points, first summarise the key messages in three or four bullet points. When these are formulated and refined, only then write them in prose. They may follow on at the end of a discussion section with or without a heading. It is a personalised statement by the author relating to the message that follows on from by the case report. Focus on the lessons that should be learnt from the case and how this could affect future clinical practice. Any conclusions may agree or disagree with current thinking and treatment modalities. If appropriate to the case, within one or two sentences describe the evidence-based lessons that may be learned. However, refrain from any speculation or unwarranted or disproportionate conclusions. 


\section{The Abstract}

The Abstracts generally follow one of two styles, narrative or structured. Consult "Instruction to Authors" in the intended journal.

A narrative abstract consists of a short version of the whole paper, usually without headings.

A structured abstract uses subheadings. These standardise the abstract and make sure that all relevant information is included. As a search engine now shows abstract, they are the basis on which most readers will decide whether to consult the complete article and cite the paper in the future.

Since they are summaries, both narrative and structured abstracts are best written on completion of the article.

Before writing the abstract in its final form, either narrative or structured, make a list of the following in "bullet form", to be switched eventually to prose:

- Introduction. In the Introduction use no more than one or two sentences to describe the case.

- Case presentation. Several sentences describe the medical history, tests and results. Conclude with a working diagnosis.

- Management and Outcome. Describe the course of the patient's treatment chronologically and any outcome measures which indicate how the patient's condition progressed through the course of management.

- Discussion. Explain both correlations and apparent inconsistencies. If fitting, in one or two sentences, describe the lessons to be learned.

An Abstract usually has a maximum of 150 words, which is much shorter than an Abstract in a research paper.

\section{The Title}

It is advisable to use a "working title" until the paper has been finalised. At that point, the definitive title can be composed, and keywords can be chosen. These two elements are best undertaken at the very end of the process of writing a manuscript as the words used MUST adequately and effectively relate to words and concepts already used and made in the body of the manuscript. Always consult the "instructions to authors" of the journal to which the manuscript is likely to be submitted for details of titles and keywords. Most journals ask for tiles of less than 15 words, and some much less.
It is good practice to add the words "A Case report" to the title as this will be seen when other authors are making a search engine search. A standard error in submitted Case report manuscripts is to add A Case report \& Literature Review.

Keywords are used when searching for the article using a search engine such as Medline. A standard list of keywords, such as MeSH (Medical subject headings) is usually in most libraries or at http://www.ncbi.nlm.nih. gov:80/entrez/meshbrowser.cgi

The use of apposite keywords is essential, and a concise list will aid others quickly accessing the paper. Again, the number of keywords is dictated by journals and usually is not more than five to six.

\section{References}

It is advisable to use a reference manager such as EndNote. Not only does it help in managing abstracts and annotated bibliographies, but it is especially useful in cases when manuscripts have been rejected by one journal and subsequently submitted to another which use a different reference system. Write references in the Vancouver style in the first instance. This is the most commonly used referencing style used in medicinal and other journals. It lists citations to studies listed in the text, by a number. At the end of the manuscript, a consecutively numbered reference list imparts details of the corresponding in-text reference.

\section{SUBMITTING A CASE REPORT}

Once the first draft has been written, check the spelling and grammar. If possible, have it proof-read by a colleague or co-author(s), and then make any final revisions.

Like most papers, case reports may require at least one or two revisions after submission to a journal. If a case report is rejected outright, submit it to other journals.

Always read the reviewers' comments, many of whom may be experts in the field, and revise the article carefully.

\section{Checklist}

- Give the patient demographics, age, sex, height, weight, race, occupation.

- Delete all patient identifiers such as date of birth, initials. 
- Describe the patient's initial complaint and present illness.

- Record the patient's medical history.

- Record the patient's family history.

- Record the patient's social history.

- Give a list of the patient's medication before admission and throughout ensuing treatment.

- List each drug's name, strength, dosage form, route, and dates of administration.

- Detail the patient's medication observance.

\section{- AVOID THESE COMMON ERRORS}

1.Photographs and radiographs must be carefully scrutinized and any possible identifiers deleted. Blocking out the eye area is often not sufficient.

2. Avoid medical abbreviations used in medical records. Write out all the information in full. In the following extract from a submitted manuscript, several errors are identifiable. The abbreviations should be written out in full: "the patient was managed for 7 days with antibiotics: Inj ceftriaxone $1 \mathrm{~g} \mathrm{BD}$, Inj Azithromycin $500 \mathrm{mg}$ OD and Tab Oseltamivir 75mg BD, oxygen therapy, intermittent non-invasive respiratory support (NIV) with a setting of $12 / 6 \mathrm{~cm}$ of water."

3. Drugs should be given either by the brand or generic name of the drug. The name MUST be followed by, in brackets, (name of the manufacturer, town and country). Brand names should be capitalised with appropriate (c) or ${ }^{\mathrm{tm}}$. Generic names are given in lowercase. In the excerpt given in (2) above, ceftriaxone, a generic name for the brand name Rocephin is correctly written in lower case but without the required name of the manufacturer, town and country in brackets. Note that azithromycin oral tablets are available as both a generic and brand-name drug. The brand name is Zithromax. In the above, it should NOT be capitalised and requires the name of the manufacturer, town and country in brackets.

The correct way is azithromycin (Manufacturer, Town, Country); alternatively, if the brand name is used, Zithromax (Pfizer Inc., New York, USA).

It is important to give details of the manufacturer as there are significant differences in formulation other than the active ingredient between manufacturers. This difference and NOT the active ingredient may play an important role and be the cause of an observed effect.

4. This also applies to instruments which may be novel or require special mention in the case.

"[...] humidity which was continuously monitored by a USB-502 logger (Measurement Computing Corp., Norton, MA, USA)."

5. All relevant results should be written in full using internationally recognised units explaining any abbreviations, e.g. "The patient was hypotensive with a blood pressure of 130/80 $\mathrm{mmHg}$ and had developed neutropenic sepsis with a white blood cell count (WCC), of 2.1 x 109/l."

6. Give details of the institute or hospital mentioned in the case, not a full address but region, town and country.

7.Provide a statement that the patient's written consent was obtained. 